\title{
On Sidon sequences of even orders
}

\author{
by \\ Sheng Chen (San Marcos, TX)
}

Let $h \geq 2$ be an integer. A set $A$ of positive integers is called a $B_{h^{-}}$ sequence if all sums $a_{1}+\ldots+a_{h}$, where $a_{i} \in A(i=1, \ldots, h)$, are distinct up to rearrangements of the summands. A $B_{h}$-sequence is also called a Sidon sequence of order $h$ [7]. Sidon was led to consider such sequences in connection with the theory of Fourier series. In [7] he raised the question of how many terms not exceeding $n$ a $B_{h}$-sequence may have. Some earlier important results on $B_{h}$-sequences may be found in [2, Ch. 2].

Let $A$ be a $B_{h}$-sequence. Denote by $A(n)$ the cardinality of $A \cap[0, n]$. For any positive integer $r$, denote by $r A$ the set of integers $\sum_{i=1}^{r} a_{i}$ where $a_{i} \in A(i=1, \ldots, r)$. It follows from the definition of $B_{h}$-sequences that

which implies

$$
h n \geq(h A)(h n) \geq\left(\begin{array}{c}
A(n) \\
h
\end{array}\right),
$$

$$
A(n)=O(\sqrt[h]{n}) .
$$

Erdős $[2,8]$ and Krückeberg [5] showed that there exists a $B_{2}$-sequence $A$ such that

$$
\limsup _{n \rightarrow \infty} \frac{A(n)}{\sqrt{n}} \geq \frac{1}{\sqrt{2}} .
$$

On the other hand, Erdős [2] also proved that, for any $B_{2}$-sequence $A$,

$$
\liminf _{n \rightarrow \infty} A(n) \sqrt{\frac{\log n}{n}}<\infty .
$$

Nash extended the result to $B_{4}$-sequences. He showed [6] that, for any $B_{4}$-sequence $A$,

$$
\liminf _{n \rightarrow \infty} A(n) \sqrt[4]{\frac{\log n}{n}}<\infty
$$

1991 Mathematics Subject Classification: Primary 11B83; Secondary 11B50, 05B10.

Key words and phrases: additive number theory, difference sets, $B_{h}$-sequence, Sidon sequences. 
A natural conjecture [4] is as follows.

Conjecture. If $A$ is a $B_{h}$-sequence, then

$$
\liminf _{n \rightarrow \infty} A(n) \sqrt[h]{\frac{\log n}{n}}<\infty .
$$

For $h$ odd, no results of this kind have been proved as far as the author knows.

For $h$ even $(h=2 k>4)$, Jia [4] showed that, if $A\left(n^{2}\right) \leq A(n)^{2}$, then

$$
\liminf _{n \rightarrow \infty} A(n) \sqrt[2 k]{\frac{\log n}{n}}<\infty .
$$

As mentioned in [4], the condition $A\left(n^{2}\right) \leq A(n)^{2}$ does not hold for all $B_{2 k}$-sequences.

The author [1] proved that a weaker result of a similar nature does hold, namely

$$
\liminf _{n \rightarrow \infty} \frac{A(n)}{\sqrt[2 k]{n}} \sqrt[4 k-4]{\log n}<\infty
$$

which has been improved by Helm [3] to

$$
\liminf _{n \rightarrow \infty} \frac{A(n)}{\sqrt[2 k]{n}} \sqrt[3 k-1]{\log n}<\infty
$$

In this paper, we give an affirmative partial answer to the Conjecture.

Theorem. For any $B_{2 k}$-sequence $A$,

$$
\liminf _{n \rightarrow \infty} A(n) \sqrt[2 k]{\frac{\log n}{n}}<\infty .
$$

Corollary. Let $A=\left\{a_{1}<a_{2}<\ldots<a_{n}<\ldots\right\}$ be an infinite $B_{2 k}$-sequence. Then

$$
\limsup _{n \rightarrow \infty} \frac{a_{n}}{n^{2 k} \log n}>0 .
$$

First some notations and lemmas.

Let $A$ be a $B_{2 k}$-sequence and $r$ be a positive integer, $1 \leq r \leq 2 k$. For any $x=x_{1}+\ldots+x_{r}$ where $x_{s} \in A(s=1, \ldots, r)$, denote by $\bar{x}$ the set $\left\{x_{1}, \ldots, x_{r}\right\}$ (counting the multiplicities of the appearance of $x_{i}$ 's) and

$$
r * A=\left\{x=x_{1}+\ldots+x_{r}: x_{s} \in A, x_{s} \neq x_{t}, 1 \leq s, t \leq r\right\} .
$$

Note that, since $A$ is a $B_{2 k}$-sequence, $x=y \in r A$ if and only if $\bar{x}=\bar{y}$.

Let $A$ be a $B_{2 k}$-sequence. Let $n$ be a large integer fixed hereafter and $u=\left\lfloor n^{1 /(2 k)}\right\rfloor$. For any sequence $B$, denote by $B_{i}(n)$, or simply $B_{i}$, the set $B \cap((i-1) k n, i k n], i=1,2, \ldots$ 
Set $D=A \cap(0, u k n], C=k * D, c_{i}=\left|C_{i}\right|$, and

$$
\tau(n)=\min _{n \leq m \leq u n} \frac{A(m)}{\sqrt[2 k]{m}}
$$

LEMMA 1.

$$
\tau(n)^{2 k} n \log n=O\left(\sum_{i=1}^{u} c_{i}^{2}\right) .
$$

Proof. Note that

$$
\left(\sum_{i=1}^{u} \frac{c_{i}}{\sqrt{i}}\right)^{2} \leq\left(\sum_{i=1}^{u} \frac{1}{i}\right)\left(\sum_{i=1}^{u} c_{i}^{2}\right) \leq 2 \log u \sum_{i=1}^{u} c_{i}^{2} .
$$

On the other hand, for any positive integer $i(1 \leq i \leq u)$,

$$
C(i k n) \geq\left(\begin{array}{c}
A(i n) \\
k
\end{array}\right) \geq c A(i n)^{k}
$$

where $c>0$ is an absolute constant depending only on $k$, and

$$
A(i n)^{k}=\left(\frac{A(i n)}{\sqrt[2 k]{i n}}\right)^{k} \sqrt{i n} \geq \tau(n)^{k} \sqrt{i n} .
$$

Hence,

$$
\begin{aligned}
\sum_{i=1}^{u} \frac{c_{i}}{\sqrt{i}} & =\sum_{i=1}^{u}\left(\frac{1}{\sqrt{i}}-\frac{1}{\sqrt{i+1}}\right) \sum_{j=1}^{i} c_{j}+\frac{1}{\sqrt{u+1}} \sum_{j=1}^{u} c_{j} \\
& \geq \frac{1}{8} \sum_{i=1}^{u} \frac{1}{i^{3 / 2}} C(i k n) \geq \frac{c}{8} \sum_{i=1}^{u} \frac{A(i n)^{k}}{i^{3 / 2}} \\
& \geq \frac{c}{8} \tau(n)^{k} \sqrt{n} \sum_{i=1}^{u} \frac{1}{i} \geq c \tau(n)^{k} \sqrt{n} \log u
\end{aligned}
$$

So,

$$
\tau(n)^{2 k} n \log u \leq O\left(\sum_{i=1}^{u} c_{i}^{2}\right) .
$$

As $u=\left\lfloor n^{1 /(2 k)}\right\rfloor$, Lemma 1 follows.

LEMMA 2.

$$
\sum_{i=1}^{u} c_{i}^{2}=O(n)
$$

Proof. Set $W=\{(x, y) \in(k * A) \times(k * A): \bar{x} \cap \bar{y}=\emptyset\}$. Define a map $f: W \rightarrow(-\infty, \infty)$ by $(x, y) \mapsto x-y$. If $f\left(x^{\prime}, y^{\prime}\right)=f(x, y)$, i.e., $x^{\prime}-y^{\prime}=x-y$, then $x^{\prime}+y=x+y^{\prime}$. As $A$ is a $B_{2 k}$-sequence, $\overline{x^{\prime}} \cup \bar{y}=\bar{x} \cup \overline{y^{\prime}}$. Since $\bar{x} \cap \bar{y}=\overline{x^{\prime}} \cap \overline{y^{\prime}}=\emptyset$, we have $x=x^{\prime}$ and $y=y^{\prime}$. Thus $f$ is one-to-one. 
For any integer $r, 1 \leq r \leq k$, let

$$
V(r, D)=\{(x, y) \in(r * D) \times(r * D):-k n<x-y<k n\} .
$$

Then

$$
\sum_{i=1}^{u} c_{i}^{2} \leq|V(k, D)|
$$

Write $V(k, D)=\bigcup_{j=0}^{k} V_{j}(k, D)$ (disjoint union) where

$$
V_{j}(k, D)=\{(x, y) \in V(k, D):|\bar{x} \cap \bar{y}|=j\}, \quad 0 \leq j \leq k .
$$

Then

$$
|V(k, D)|=\sum_{j=0}^{k}\left|V_{j}(k, D)\right| .
$$

For any $B_{2 k}$-sequence $B$ and any integers $j, r(0 \leq j, r \leq k)$, let $W(r, D, j, B)$ be the set of 4 -tuples $(x, b, b, y)$ such that

(a) $x, y \in r * D$ with $-k n<x-y<k n$,

(b) $b \in j * B$, and

(c) $\bar{b} \cap \bar{x}=\bar{b} \cap \bar{y}=\bar{x} \cap \bar{y}=\emptyset$.

If $j=0$, we simply write $W(r, D)$ instead.

Note that $\left|V_{j}(k, D)\right|=O(|W(k-j, D, j, D)|)$. Combining this with (1) and (2), we have

$$
\sum_{i=1}^{u} c_{i}^{2}=O\left(\sum_{j=0}^{k}|W(k-j, D, j, D)|\right) .
$$

Hence it suffices to show that, for all $j(0 \leq j \leq k)$,

$$
|W(k-j, D, j, D)|=O(n) .
$$

Case $j=0$. Then $W(k, D, 0, D)=W(k, D) \subseteq W$ and $f(W(k, D)) \subseteq$ $(-k n, k n)$. Hence,

$$
|W(k, D, 0, D)| \leq 2 k n \text {. }
$$

Case $j=k$. Then $V(0, D, k, D)=\{(b, b): b \in k * D\}$. Hence,

(5) $|W(0, D, k, D)|=|k * D| \leq|D|^{k}=A(u k n)^{k}=O\left((\sqrt[2 k]{u k n})^{k}\right)=O(n)$.

Case $1 \leq j \leq k-1$. Let $I=\left\{i: 1 \leq i \leq u\right.$ and $\left.\left|D_{i}\right| \geq 2 k\right\}$. Set $B=\bigcup_{i \in I} D_{i}$, and $B^{\prime}=D \backslash B$. We divide our proof into two subcases.

Hence,

Subcase I: $|B| \leq\left|B^{\prime}\right|$. Note $B^{\prime}=\sum_{1 \leq i \leq u ; i \notin I} D_{i}$. So $\left|B^{\prime}\right| \leq u(2 k-1)$.

$$
|D|=\left|B^{\prime}\right|+|B| \leq 2\left|B^{\prime}\right| \leq 4 k u .
$$


Therefore,

(6) $|W(k-j, D, j, D)| \leq|(2 k-j) * D| \leq|D|^{2 k-j} \leq(4 k u)^{2 k-j}=O(n)$.

Subcase II: $\left|B^{\prime}\right| \leq|B|$. Note $B$ is also an $B_{2 k}$-sequence. We claim

$$
|W(k-j, D, j, B)|=O(n) .
$$

To show (7), define a map $v: W(k-j, D, j, B) \rightarrow W$ as follows. Let $(x, b, b, y) \in W(k-j, D, j, B)$. As $b \in j * B, b=\sum_{s=1}^{j} b_{s}$ where $b_{s} \in D_{i_{s}}$ and $i_{s} \in I(s=1, \ldots j)$. Since $\left|D_{i_{s}}\right| \geq 2 k$, we can choose $b_{s}^{\prime} \in D_{i_{s}}(s=1, \ldots, j)$ so that

(i) all $b_{s}^{\prime}$ 's are distinct and

(ii) $b_{s}^{\prime} \notin \bar{x} \cup \bar{y} \cup \bar{b}, s=1, \ldots, j$.

Let $b^{\prime}=\sum_{s=1}^{j} b_{s}^{\prime}$. Then $\left(x, b, b^{\prime}, y\right) \in W$. Define $v: W(k-j, D, j, D) \rightarrow$ $W$ by $(x, b, b, y) \mapsto\left(x, b, b^{\prime}, y\right)$. Clearly, $v$ is well defined and one-to-one. Furthermore, by the choice of $b_{s}^{\prime},-k n \leq b_{s}-b_{s}^{\prime} \leq k n$ for all $1 \leq s \leq j$. So, $-j k n \leq b-b^{\prime} \leq j k n$. Hence,

$$
-(j+1) k n<(x+b)-\left(b^{\prime}+y\right)<j k n+k n .
$$

Thus,

$$
|W(k-j, D, j, B)|=|f(v(W(k-j, D, j, B)))| \leq 2(j+1) k n \leq 2 k^{2} n,
$$

which is $(7)$.

Note

(8) $W(k-j, D, j, D)$

$$
\begin{aligned}
& =\bigcup_{(x, y) \in W(k-j, D)}\{(x, b, b, y): b \in j * D, \bar{b} \cap(\bar{x} \cup \bar{y})=\emptyset\} \\
& =\bigcup_{(x, y) \in W(k-j, D)}\{(x, b, b, y): b \in j *(D \backslash(\bar{x} \cup \bar{y}))\},
\end{aligned}
$$

and similarly,

(9) $W(k-j, D, j, B)=\bigcup_{(x, y) \in W(k-j, D)}\{(x, b, b, y): b \in j *(B \backslash(\bar{x} \cup \bar{y}))\}$.

On the other hand, for any $z=(x, y) \in W(k-j, D)$, it follows from the assumption that $\left|B^{\prime}\right| \leq|B|$, and

$$
|D \backslash \bar{z}|=|(B \backslash \bar{z})|+\left|B^{\prime} \backslash \bar{z}\right| \leq 2|B \backslash \bar{z}|+|z| \leq 2|B \backslash \bar{z}|+2 k .
$$

Therefore,

(10) $|j *(D \backslash \bar{z})|=\left(\begin{array}{c}|D \backslash \bar{z}| \\ j\end{array}\right) \leq\left(\begin{array}{c}2|B \backslash \bar{z}|+2 k \\ j\end{array}\right) \leq c(|j *(B \backslash \bar{z})|+1)$,

where $c$ is an absolute constant depending only on $j$, hence only on $k$. 
Combining (7)-(10), we have

$$
\text { (11) } \begin{aligned}
|W(k-j, D, j, D)| & =\sum_{z \in W(k-j, D)}|j *(D \backslash \bar{z})| \\
& \leq \sum_{z \in W(k-j, D)} c(|j *(B \backslash \bar{z})|+1) \\
& \leq c\left(\sum_{z \in W(k-j, D)}|j *(B \backslash \bar{z})|+\sum_{z \in W(k-j, D)} 1\right) \\
& =c(|W(k-j, D, j, B)|+|W(k-j, D)|)=O(n) .
\end{aligned}
$$

Now Lemma 2 follows from (3)-(5) and (11).

Proof of the Theorem. It follows from Lemmas 1 and 2 that $\tau(n)^{2 k} \log n=O(1)$. Hence,

$$
\begin{aligned}
\liminf _{n \rightarrow \infty} A(n) \sqrt[2 k]{\frac{\log n}{n}} & =\liminf _{n \rightarrow \infty} \inf _{n \leq m \leq u n}\left(A(m) \sqrt[2 k]{\frac{\log m}{m}}\right) \\
& \leq \liminf _{n \rightarrow \infty} \inf _{n \leq m \leq u n}\left(\frac{A(m)}{\sqrt[2 k]{m}} \sqrt[2 k]{\log (u n)}\right) \\
& \leq 2 \liminf _{n \rightarrow \infty} \tau(n) \sqrt[2 k]{\log n}<\infty .
\end{aligned}
$$

Acknowledgments. The author would like to thank Weizhen Gu for a helpful discussion.

\section{References}

[1] S. Chen, A note on $B_{2 k}$-sequences, preprint.

[2] H. Halberstam and K. F. Roth, Sequences, Springer, New York 1983.

[3] M. Helm, A remark on $B_{2 k}$-sequences, preprint.

[4] X.-D. Jia, On $B_{2 k}$-sequences, J. Number Theory, to appear.

[5] F. Kr ückeberg, $B_{2}$-Folgen und verwandte Zahlenfolgen, J. Reine Angew. Math. 206 (1961), 53-60.

[6] J. C. M. Nash, On B-sequences, Canad. Math. Bull. 32 (1989), 446-449.

[7] S. Sidon, Ein Satz über trigonometrische Polynome und seine Anwendungen in der Theorie der Fourier-Reihen, Math. Ann. 106 (1932), 536-539.

[8] A. Stöhr, Gelöste und ungelöste Fragen über Basen der natürlichen Zahlenreihe. II, J. Reine Angew. Math. 194 (1955), 111-140.

DEPARTMENT OF MATHEMATICS

SOUTHWEST TEXAS STATE UNIVERSITY

SAN MARCOS, TEXAS 78666

U.S.A.

E-mail: SC03@SWTEXAS.BITNET 